\title{
RISCOS PSICOSSOCIAIS NO TRABALHO: CONCEITOS, VARIÁVEIS E INSTRUMENTOS DE PESQUISA
}

\author{
Aline Jacinto \\ Suzana da Rosa Tolfo \\ (Universidade Federal de Santa Catarina - UFSC)
}

\begin{abstract}
Resumo
$\mathrm{O}$ artigo apresenta uma revisão da literatura sobre riscos psicossociais no trabalho, explorando as principais teorias para definir os mesmos. Os riscos psicossociais no trabalho passaram a ser tema de interesse de estudos na Europa de forma mais acentuada, a partir do ano de 2000, haja vista os altos índices de suicídios nas empresas europeias. Apesar de existirem diversos estudos recentes sobre os riscos psicossociais no trabalho, não há uma única teoria para explicá-lo. Há duas principais vertentes teóricas que sustentam a temática dos riscos psicossociais, entre elas: a Psicodinâmica do Trabalho e as Teorias de Estresse. Constatou-se que algumas variáveis são privilegiadas nos diferentes modelos teóricos referentes aos riscos psicossociais no trabalho.
\end{abstract}

Palavras-chave: Fatores psicossociais; fatores de risco; trabalho.

\section{Abstract \\ Psychosocial risks at work: concepts, variables and research instruments}

The article presents a review of the literature on psychosocial risks at work, exploring the main theories to define them. Psychosocial risks at work became a major topic of study in Europe in the year 2000, given the high rates of suicide in European companies. Although there are several recent studies on psychosocial risks at work, there is no single theory to explain it. There are two main theoretical aspects that support the theme of psychosocial risks, among them: Work Psychodynamics and Stress Theories. It was verified that some variables are privileged in the different theoretical models concerning the psychosocial risks at work.

Keywords: Psychosocial factors; risk factors; job.

\section{Introdução}

Assim como a sociedade, os locais e práticas de trabalho e os processos de produção e prestação de serviços têm sofrido constantes mudanças, devido às novas tecnologias, às condições sociais e econômicas variáveis. Estas situações contemporâneas do contexto laboral acarretam em desafios e riscos, novos e emergentes. Assim, as relações trabalho- 
saúde têm exigido abordagens técnicas, administrativas e políticas para garantir segurança e saúde no trabalho (Agência Europeia para a Segurança e Saúde no Trabalho, 2010).

Segundo Costa e Santos (2013) essas mudanças no mundo do trabalho como as alterações do valor e do significado do trabalho, as inovações tecnológicas, as novas formas de organização, as modificações dos espaços, do conteúdo e da natureza do trabalho, têm ocorrido em função das exigências do mercado por organizações dinâmicas, facilmente adaptáveis a um ambiente exigente e mutável. As modificações sobrevindas no mundo do trabalho acarretaram em urgências por maior produtividade, pressões no trabalho, aumento da complexidade das atividades, relações de trabalho tensas e precárias, expectativas irrealizáveis, fadiga e esgotamento profissional, estes constituem-se como fatores psicossociais de risco no trabalho, os quais podem ocasionar consequências diretas nas condições de saúde dos trabalhadores, como incapacidade laborativa, adoecimentos, e repercussões na vida social e familiar (Guimarães, 2013; Serafim e outros, 2012).
Um dos desafios para a segurança e saúde no trabalho implica em reconhecer os riscos psicossociais, perceber qual seu impacto na saúde dos trabalhadores, bem como formas de intervir nas situações de trabalho, a fim de criar condições que permitam uma gestão visando melhorar a saúde, a segurança e o bem-estar (Costa \& Santos, 2013). Segundo os autores citados anteriormente, o interesse pela temática difundiu-se massivamente na Europa a partir do ano de 2000, em função dos acontecimentos de suicídios nas grandes empresas europeias da época, os quais foram atribuídos a situações de trabalho. Hirata (2011) afirma que em empresas francesas como a France Telecóm, a Renault e a Peugeot, o número de suicídios tem aumentado nos últimos anos devido ao trabalho intensificado e sob pressão, às práticas de assédio moral, à falta de solidariedade e de trabalho em equipe.

Para compreender melhor sobre o termo "psicossocial" é importante mencionar que se refere à interação entre indivíduo (psico) e seu entorno social (Ministerio de Empleo y Seguridad Social de la España, 2012). Álvarez (2012) define que os fatores psicossociais podem ser tanto favoráveis como de riscos. São considerados favoráveis quando contribuem para o desenvolvimento da atividade laboral e favorecem a qualidade 
de vida das pessoas, as relações de trabalho e a produtividade. Mas, os fatores psicossociais se convertem em risco quando têm o potencial de causar danos psicológico, físico e social aos indivíduos e são fontes diretas do estresse ocupacional.

Apesar de existirem estudos recentes sobre os riscos psicossociais no trabalho, a complexidade da origem de seus fatores e as particularidades dos contextos nos quais se manifestam têm apresentado dificuldades consideráveis nos processos de especificação, diagnóstico, prevenção e intervenção para profissionais que atuam com saúde e segurança nas organizações (Borges, Guimarães \& Silva, 2013). Em meio as diferentes formas de compreensão dos riscos psicossociais no trabalho, levanta-se um questionamento: quais variáveis vem sendo privilegiadas nos diferentes modelos teóricos sobre riscos psicossociais no trabalho? Buscouse com este artigo, contribuir de forma exploratória para a problematização teórica deste fenômeno.

\section{Modelos teóricos para}

compreensão dos riscos psicossociais no trabalho

Gollac e Bodier (2011) do Colégio de Expertise em monitoramento de riscos psicossociais no trabalho da França, afirmam que não há uma definição única para riscos psicossociais. Para os autores, riscos psicossociais são riscos para a saúde criados pelo trabalho por meio de mecanismos sociais e psíquicos, como as condições de trabalho, fatores organizacionais e relacionais, manifestando-se na saúde mental, social e física. Assim, para considerar um risco no trabalho como psicossocial, entende-se que sua origem é de ordem psicológica e social, e sua manifestação pode ser de ordem psicológica, social e física.

Uma das principais correntes teóricas para a compreensão dos riscos psicossociais no trabalho, a Psicodinâmica do Trabalho dá ênfase na centralidade do trabalho na vida das pessoas, analisando aspectos deste trabalho os quais podem favorecer a saúde ou a doença (Glina, Rocha, Batista, \& Mendonça, 2001). Mendes (2007) descreve que o objeto de estudo da Psicodinâmica do Trabalho são as relações dinâmicas entre processos de subjetivação e organização do trabalho. Estas relações se manifestam nas vivências de prazer-sofrimento, nas estratégias de ação para mediações de contradições na organização do trabalho, nas patologias sociais, no adoecimento e na saúde. 
Outro modelo de explicação para a compreensão dos riscos psicossociais no trabalho é por meio das teorias do estresse, o qual será explicado o porquê de seu uso por meio de documento do Ministério do Emprego e Segurança Social da Espanha (2012). Os fatores de riscos psicossociais são aspectos da organização e seu ambiente social, sendo eles carga e ritmo de trabalho excessivos, comunicações empobrecidas, falta de apoio, trabalho em turnos, falta de participação das decisões, tarefas desagradáveis, conflitos nas relações interpessoais no trabalho, conflitos entre as exigências domésticas e as do trabalho. Estes fatores podem levar o indivíduo ao estresse, que por sua vez é considerado um risco psicossocial ao trabalhador, pois pode ocasionar em danos à sua saúde, como físicos (problemas cardiovasculares e gastrointestinais), psíquicos (transtornos de ajustamento e depressão) e comportamentais (dependência ou vício). Ressalte-se, que o estresse não é uma doença, mas uma situação que pode acarretar danos à saúde, como os mencionados anteriormente, assim como, ocasionar o burnout, o qual é uma situação de desgaste ou estresse crônico (Ministerio de Empleo y Seguridad Social de la España, 2012).

Para Costa e Santos (2013) os fatores psicossociais de risco no trabalho são muitas vezes caracterizados como fatores estressores de trabalho, portanto, a maior visibilidade do tema riscos psicossociais no trabalho é compreendida como uma extensão do termo estresse. Visto a importância desses modelos teóricos para a compreensão dos riscos psicossociais no trabalho, em seguida serão apresentadas as teorias: Psicodinâmica do Trabalho e algumas teorias do estresse como Modelo Estresse/ Apoio e Saúde Mental; Modelo Desequilíbrio EsforçoRecompensa e Modelo Demanda-Controle, mas para Papaioannou e Billias (2012) as duas últimas são as mais utilizadas para explicar os riscos psicossociais no trabalho.

\section{Psicodinâmica do Trabalho}

A Psicodinâmica do Trabalho foi desenvolvida na França por Christophe Dejours nos anos de 1990. Suas bases conceituais são elaboradas a partir da análise da dinâmica inerente a determinados contextos de trabalho, caracterizada pela atuação de forças, visíveis e invisíveis, objetivas e subjetivas, psíquicas, sociais, políticas e econômicas que podem ou não deteriorar esse contexto, transformando-o em lugar de saúde e/ou de patologias e de adoecimento (Mendes, 2007). 
Brant e Minayo-Gomes (2004) afirmam que a psicodinâmica do trabalho tem como pressuposto que o sofrimento no trabalho pode ser transformado em adoecimento do trabalhador. Para Dejours (2004) não há como separar o "dentro do trabalho" e "fora do trabalho", pois afirma que o trabalho é central na vida do sujeito, havendo continuidade entre 0 funcionamento psíquico no trabalho e fora dele.

Este campo de estudo prioriza os fatores relacionados à organização do trabalho, como ritmo, jornada, hierarquia, responsabilidade, controle, assim como às condições de trabalho, a qual está relacionada às pressões físicas, biológicas, mecânicas e químicas do posto de trabalho. Estas pressões podem acarretar em danos o corpo dos trabalhadores, os quais podem originar desgaste, doenças somáticas e envelhecimento (Dejours \& Abdoucheli, 1993).

A proposta dejouriana tem como método principal as técnicas qualitativas, como a escuta, a interpretação e a devolução, privilegiando a entrevista coletiva, pois nesta ocorre interação grupal permitindo reconstruir a lógica das pressões no trabalho e as defesas coletivas contra os efeitos psicológicos dessas pressões (Dejours \& Abdoucheli, 1993).
Apesar da Psicodinâmica do Trabalho ser contrária ao uso de questionários e estudos epidemiológicos, é possível encontrar estudos brasileiros sobre riscos psicossociais no trabalho, sob esta abordagem se utilizando de técnicas quantitativas, geralmente estes estudos fazem uso do Inventário de Trabalho e Riscos de Adoecimento (ITRA). Este instrumento brasileiro, foi desenvolvido por Mendes e Ferreira (2007), sendo composto por quatro escalas, as quais avaliam as seguintes dimensões e seus respectivos fatores:

- Escala de Avaliação do Contexto de Trabalho (EACT): organização do trabalho, condições de trabalho e relações socioprofissionais;

- Escala de Custo Humano do Trabalho (ECHT): custo físico, cognitivo e afetivo;

- Escala de Indicadores de PrazerSofrimento no Trabalho (EIPST): prazer (realização socioprofissional e liberdade de expressão) e sofrimento (falta de reconhecimento e esgotamento profissional);

- Escala de Avaliação dos Danos Relacionados ao Trabalho (EADRT): danos físicos, psicológicos e sociais.

Modelo Estressel Apoio e Saúde Mental 
O modelo de teoria do estresse proposto por Ostermann (1989, citado por Areias \& Guimarães, 2004) tem como pressuposto teórico que a saúde mental e os "fatores psicossociais de risco" (nomenclatura utilizada pelo autor) são determinados por fatores de apoio, bem como por estressores nas dimensões pessoal (self), social e de trabalho. De acordo com a teoria, cada uma das dimensões pode contribuir de maneira positiva (como apoio) ou negativa (como estressor) nas condições gerais da vida, sendo que os fatores de apoio podem anular os efeitos danosos do estresse. $\mathrm{Na}$ Tabela 1 são demonstrados as dimensões pessoal, social e trabalho, bem como seus respectivos fatores de estresse e fatores de apoio.

Tabela 1

Dimensões e seus fatores estressores e de apoio

\begin{tabular}{llll}
\hline Dimensões & Fatores de estresse & Fatores de apoio \\
\hline Pessoal (Self) & Disposição individual e comportamental & Disposição & individual \\
& como emoções e características de & comportamental compatível com \\
& personalidade. & atitudes de autoconfiança, coping ou \\
& habilidades para manejar e controlar \\
& eventos de vida, assim como ter \\
& perspectivas congruentes com seus \\
& dados de realidade.
\end{tabular}

\begin{tabular}{|c|c|c|}
\hline Social & $\begin{array}{l}\text { Situações que acontecem fora do âmbito } \\
\text { do trabalho, como condições e vida } \\
\text { caóticas, perigosas ou insalubres, } \\
\text { relações de conflito com familiares, } \\
\text { amigos e vizinhos, e responsabilidades } \\
\text { que envolvem tensões. }\end{array}$ & $\begin{array}{l}\text { Relações satisfatórias e condições de } \\
\text { vida confortáveis e protegidas, assim } \\
\text { como apoio de familiares e amigos, } \\
\text { permitindo conforto psicológico e } \\
\text { físico à pessoa que vivencia uma } \\
\text { situação estressante. }\end{array}$ \\
\hline Trabalho & $\begin{array}{l}\text { Fatores de estresse de trabalho gerados } \\
\text { pela natureza do trabalho e suas } \\
\text { condições, pelos conflitos interpessoais } \\
\text { entre o trabalho e as obrigações pessoais } \\
\text { e pela necessidade de autorrealização. }\end{array}$ & $\begin{array}{l}\text { Condições de trabalho confortáveis, } \\
\text { que propiciam o desenvolvimento de } \\
\text { potencialidades, trabalho com alta } \\
\text { significação, boas relações } \\
\text { interpessoais e de apoio em geral. }\end{array}$ \\
\hline
\end{tabular}

Tabela elaborada com base em Ostermann (1989, citado por Areias \& Guimarães, 2004). 
Para Ostermann (1989, citado por Areias \& Guimarães, 2004) a definição operacional do termo fatores psicossociais de risco resulta do aumento de fatores de estresse e a diminuição do apoio presentes no âmbito pessoal, na vida social e no trabalho. Este desequilíbrio pode ocasionar sentimento de mal-estar e dificuldade em lidar consigo mesmo. Porém quando há um maior número de fatores de apoio do que de fatores de estresse nas três dimensões citadas, resulta em bem estar e capacidade de enfrentar a realidade de forma produtiva, assim tem-se a definição de saúde mental.

Osterman criou um Questionário chamado SWS (Self, Workand Social) o qual foi validado para a população brasileira por Guimarães e MacFadden (1999). Este instrumento possui 184 itens, distribuídos entre oito escalas: 1- Fatores Psicossociais de Risco, 2- Saúde Mental,

\section{Modelo Desequilíbrio Esforço-}

$$
\text { Recompensa-ERI }
$$

O modelo proposto por Siegrist (1996) tem como foco o desequilíbrio entre os esforços despendidos pelo sujeito e as recompensas recebidas. Este modelo teórico está pautado na noção de
3- Estresse Social, 4- Apoio Social, 5Estresse no Trabalho, 6- Apoio no Trabalho, 7- Estresse Pessoal, 8- Apoio Pessoal.

O estudo de Guimarães e Areias (2004) utilizou o Questionário SWS como instrumento de coleta de dados com 400 trabalhadores de uma Universidade Pública. Os resultados demonstraram que o gênero feminino apresentou mais fatores psicossociais de risco, estresse no trabalho, estrese social e estresse pessoal. Por outro lado, o gênero masculino apresentou melhores resultados em saúde mental e fatores de apoio (trabalho, social e pessoal). Desta forma, percebeu-se que os Fatores Psicossociais de Risco estão fortemente relacionados com os fatores estressores, bem como a Saúde Mental está fortemente relacionada com fatores de apoio, ou seja, os fatores de Apoio podem anular os fatores de Estresse.

reciprocidade social, a qual é caracterizada por investimentos mútuos de cooperação com base na norma da expectativa de retorno, onde os esforços são equalizados pelas respectivas recompensas (Siegrist, 2012). O autor afirma que a não reciprocidade, ou seja, altos esforços despendidos e baixas recompensas, é suscetível a provocar emoções negativas e 
experiências de estresse, as quais podem gerar efeitos adversos à saúde. Por outro lado, recompensas sociais adequadas podem evocar emoções positivas e promover saúde e bem estar.

Uma grande especificação desta abordagem diz respeito ao papel do trabalho, para o qual a maioria das pesquisas em ERI tem se voltado. De acordo com a teoria, os esforços no trabalho podem ser físicos ou psicológicos, já as recompensas podem ser de diferentes ordens: salário, estima e oportunidades de carreira, incluindo a segurança no emprego (Siegrist, 2012). O autor afirma que o desequilíbrio entre alto esforço e baixa recompensa, acontece sobre as seguintes condições:

1- os contratos de trabalho são mal definidos ou faltam alternativas para o trabalhador (por exemplo, devido à falta de mobilidade, baixo nível de habilidade, mercado de trabalho precário);

2- o trabalhador pode aceitar esse desequilíbrio por estratégia para melhorar as perspectivas futuras de trabalho;

3- as características do trabalhador podem contribuir para o cenário de desequilíbrio, como comprometimento excessivo (necessidade de aprovação, competitividade, dificuldade em se afastar das obrigações do trabalho quando se está fora dele).
Siegrist (2012) aborda que esforço elevado e baixa recompensa aumentam os riscos à saúde, assim como trabalhadores com comprometimento excessivo possuem maior risco. Mas quando estas duas condições estão presentes, o risco para a saúde é ainda maior. Desta maneira, para investigar estes fatores, Siegrist (1996) criou a Escala Effort-Reward Imbalance ERI, a qual foi adaptada para a população brasileira por Silva e Barreto (2010). O instrumento é composto por 23 itens, os quais avaliam três dimensões: Esforço, Recompensa e Comprometimento Excessivo.

\section{Modelo Demanda-Controle}

O estresse está entre os processos relativos ao contínuo saúde-doença que têm despertado grande interesse de pesquisadores e de profissionais de diversas áreas, dentre as quais a psicologia das organizações e do trabalho. Inicialmente as teorias do estresse foram desenvolvidas para descrever reações de estresse agudo e inevitável em situações que ameaçavam a sobrevivência. Mas o modelo Demanda/Controle, ou Job Strain Model, foi desenvolvido considerando que no ambiente psicossocial de trabalho os "fatores estressores" são crônicos, inicialmente não apresentam ameaça à 
vida, mas podem se tornar riscos potencias à saúde do trabalhador (Karasek, 1979; 1998), e estes riscos também são chamados de riscos psicossociais.

Este modelo teórico analisa as situações de trabalho relacionadas às características psicossociais que funcionam como predispositoras ao risco de adoecimento. Tais características são demandas psicológicas combinadas ao controle exercido sobre o trabalho (Karasek, 1998). De acordo com Araújo, Graça, e Araújo (2003), “a partir da combinação dessas duas dimensões, o modelo distingue situações de trabalho específicas que, por sua vez, estruturam riscos diferenciados à saúde" (p. 992).

Para Karasek (1998) a dimensão demanda psicológica além de se referir ao "quanto se trabalha", envolve situações de trabalho às quais são exigidos do trabalhador, concentração, ritmo, volume, tempo para realização das tarefas, demandas conflitantes, carga de trabalho. Esta última se refere às exigências do trabalho e é considerada a componente central de demandas psicológicas relativas ao trabalho para a maioria dos trabalhadores. O autor alerta para essa problemática nas jornadas de trabalhos em

turnos, especialmente nos rotativos, os quais apresentam demasiada exigência e mostram-se associados a problemas sociais e ao aumento de doenças. Apesar de um nível elevado de exigências ser prejudicial à saúde, é importante um nível moderado de desafios para o trabalhador aprender coisas novas e ter um rendimento eficaz.

Por seu lado, a dimensão controle envolve dois componentes, a autoridade decisória e o uso de habilidades. O primeiro compreende o grau de habilidade individual para tomadas de decisão sobre o próprio trabalho, bem como a influência no grupo de trabalho e na política de gestão. Com relação ao uso de habilidades, este refere-se ao grau de aprendizagem de coisas novas no trabalho, criatividade, repetitividade, tarefas variadas e desenvolvimento de habilidades especiais individuais. (Karasek, 1979).

Cada uma das duas dimensões são compostas por fatores psicossociais, os quais podem ser favoráveis ou de risco. Segundo Karasek (1998), a interação das duas dimensões, demanda e controle, podem gerar quatro experiências psicológicas no trabalho, conforme Figura 1. 
Figura 1: Modelo Demanda/Controle

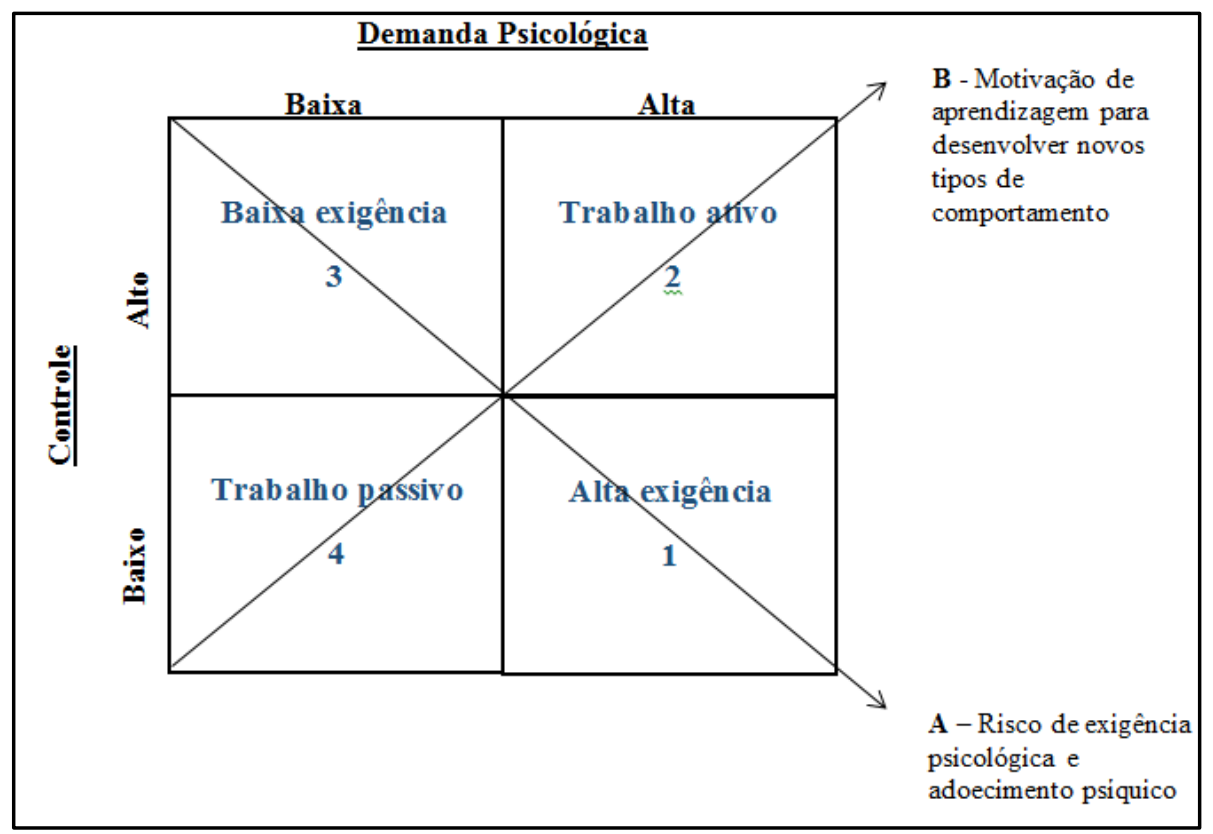

Fonte: Karasek (1979).

As referidas experiências, também chamadas de quadrantes são:

1- Alta exigência: acontece quando há alta demanda psicológica e baixo controle, neste quadrante há maior exposição de riscos à saúde física e mental, portanto é considerado o mais nocivo à saúde do trabalhador. Exemplos de situações de alta exigência: trabalhadores de uma facção que estão submetidos a forte pressão de prazo para entrega sob ameaça de demissão, quando não conseguem adotar medidas necessárias para lidar com a pressão, apresentam sintomas graves de tensão. Outro exemplo é quando o ritmo do trabalho é impresso por máquinas, onde o trabalhador é obrigado à segui-las, como os trabalhadores de montadoras, os cortadores, os inspetores e os estivadores de porto, bem como garçons e cozinheiros (Karasek, 1998).

2- Trabalho ativo: remete a alta demanda (mas não excessiva) e alto controle, apresenta probabilidade de melhor saúde psíquica, pois apesar das exigências do trabalho serem elevadas, há um alto controle sobre as tarefas, possibilitando maior aprendizagem e crescimento do trabalhador, e consequentemente maior produtividade. Pode-se citar algumas profissões com esta característica, como advogados, juízes, 
gestores, médicos, enfermeiros e engenheiros (Karasek, 1998).

3- Baixa exigência: ocorre quando a demanda é baixa e o controle é elevado, assim o ritmo de trabalho é impresso pelo próprio trabalhador. Profissões que se enquadram em baixa exigência são eletricistas, fornecedores, pessoas que atuam com reparos, pois tem a possibilidade de marcar seu próprio ritmo de trabalho (Karasek, 1998).

4- Trabalho passivo: apresenta baixa demanda e baixo controle; pode gerar problemas nocivos à saúde à medida que produz um contexto de trabalho pouco motivador, pois gera declínio da aprendizagem e perda progressiva das habilidades adquiridas anteriormente. As profissões que possuem esta característica incluem contadores, trabalhadores administrativos, de lojas, de empresas de transporte e serviços com baixo nível de escolaridade como faxineiros (Karasek, 1998).

Os quatro quadrantes podem ser representados por duas diagonais, A e B. A diagonal A representa risco de distúrbios de ordem psicológica e doença física e seu quadrante mais nocivo está representado pelo 1- alta exigência. Com relação a diagonal $\mathrm{B}$, encontra-se maior motivação para desenvolver novas diretrizes de comportamento. Porém o quarto quadrante (trabalho passivo) o qual faz parte desta diagonal, pode ser prejudicial à saúde do trabalhador à medida que pode proporcionar um declínio na atividade global do trabalhador, assim como uma redução da capacidade de produção de solução para as atividades e problemas enfrentados (Karasek, 1998; Araújo e outros, 2003). Desta maneira percebe-se que os quadrantes com mais chances de acarretar em adoecimento ao trabalhador, são combinações as quais o controle aparece baixo.

Considerando que controle sobre o trabalho pode não ser a única fonte disponível para o enfrentamento das demandas do trabalho, grande número de estudos sugere que suporte social pode funcionar como moderador da demanda dentro e fora do trabalho. (Araújo, 1999, p. 54).

Esta dimensão foi adicionada ao modelo teórico posteriormente por (Karasek \& Theorell, 1990).

São considerados suporte social o suporte da chefia e o suporte dos colegas de trabalho, os quais apesar de serem de natureza coletiva, podem modificar as dimensões de ordem individual da relação 
demanda-controle e a saúde (Karasek, 1998).

Karasek (1998) demonstra a comprovação empírica da hipótese em diversos estudos, segundo o qual trabalhos com alta demanda e baixo controle (quadrante alta exigência) somados ao baixo apoio social, apresentaram maiores riscos de adoecimento. Desta forma, o apoio social funciona como um fator de proteção contra os efeitos nocivos do estresse. De acordo com Araújo (1999) além do suporte social foi incluída outra dimensão, a demanda física, pois elevado esforço físico pode resultar em esforço psicológico.

Para a mensuração dos fatores psicossociais no trabalho, os quais podem ser de risco ou de proteção, foi desenvolvido o Job Content Questionnaire (JCQ) por Karasek (1985). Este instrumento, o qual possui versão brasileira é composto por 49 itens, os quais estão distribuídos nas seguintes dimensões: Demanda psicológica, Demanda física, Controle sobre o trabalho, Suporte social, Insegurança no trabalho. Há também uma versão resumida do JCQ, o Job Stress Scale (JSS) elaborada por Theörel na Suécia e validada para o Brasil por Alves, Chor, Faersten, Lopes e Werneck (2004). Este instrumento possui 17 itens, os quais avaliam demanda, controle e suporte social.

Vale mencionar o Copenhagen Psychosocial Questionnaire (CoPsoQ), instrumento criado na Dinamarca por Kristensen e Borg no ano de 2000, para avaliar os riscos psicossociais no trabalho. Seu enquadramento teórico possui uma perspectiva a qual cobre ampla gama de aspetos e conceitos. Este instrumento se utiliza de diferentes modelos teóricos, dentre eles Demanda-Controle e Desequilíbrio Esforço-Recompensa. O CoPsoQ, instrumento de referência em instituições internacionais, é utilizado para fins de investigação, avaliação e prevenção dos riscos psicossociais no trabalho. Este questionário foi adaptado para o contexto espanhol, passando a se chamar CoPsoQ ISTAS 21. O instrumento é utilizado por pequenas, médias e grandes empresas na Espanha, com o intuito de identificar os fatores de riscos psicossociais no trabalho e preveni-los. O CoPsoQ ISTAS 21 possui 20 itens que avaliam: exigências psicológicas, dupla presença (exigências domésticas), controle sobre o trabalho, apoio social e qualidade de liderança, e recompensas pelo trabalho (Ministerio de Empleo y Seguridad Social de la España, 2012; Silva, 2006).

De acordo com Karasek (1998) esta teoria se torna importante ao ajudar a 
entender claramente questões pertinentes para análise da saúde e segurança no trabalho, como:

- As características da organização social do trabalho causam doenças e lesões, e não apenas os perigos físicos;

- As consequências relacionadas ao estresse tem relação com a organização social da atividade do trabalho e não somente com suas exigências;

- A atividade social do trabalho afeta os riscos relacionados ao estresse, não somente as características pessoais;

- As possibilidades "estresse positivo" e "estresse negativo" podem ser explicados em termos de combinações de demanda e de controle.

\section{Considerações Finais}

Percebe-se a importância de se investigar os riscos psicossociais no trabalho, pois os mesmos acarretam em danos não somente ao trabalhador, como também à organização. Independentemente do modelo teórico utilizado, estudos nacionais e internacionais tem demonstrado a eficácia das teorias abordadas neste artigo para compreender riscos psicossociais no trabalho.
Foi possível verificar as aproximações das diferentes teorias, no que diz respeito aos conceitos dos fatores psicossociais de risco. Verificou-se que a variável relações interpessoais vêm sendo privilegiada nos diferentes modelos teóricos, sendo que na teoria DemandaControle é denominada de "Suporte Social", pela Psicodinâmica do Trabalho de "Relações Socioprofissionais", pelo Modelo Estresse/Apoio e Saúde Mental é chamada de "Apoio no Trabalho". A Demanda psicológica e Demanda física as quais fazem parte da teoria DemandaControle, são denominadas de "Condições de trabalho" pela Psicodinâmica do Trabalho. Quanto ao controle sobre o trabalho, o qual é composto por vários fatores de acordo com a teoria DemandaControle (como autonomia, uso de habilidades, participação nas decisões, opinar sobre o trabalho) também é considerado como importante de ser investigado pela Psicodinâmica do Trabalho, porém nesta é chamado de "Liberdade de Expressão".

É preciso avançar para que os riscos psicossociais nas organizações sejam prevenidos, e para que possa haver além de prevenção de adoecimento e acidentes de trabalho, a promoção em saúde. Para tanto, é importante o 
engajamento de equipes multidisciplinares, as quais possam contar com psicólogos, profissionais de segurança, engenheiros, médicos, enfermeiros, educadores físicos. Sob o olhar destes profissionais é possível ter uma visão global dos trabalhadores, e consequentemente, desenvolver ações eficientes que visem a saúde e a segurança dos trabalhadores.

\section{Referências}

Agência Europeia para a Segurança e Saúde no Trabalho (2010). Inquérito europeu das empresas de riscos novos e emergentes (ESENER). Disponível em: http://osha.europa.eu/pt/publications/reports/pt_esener1-summary.pdf/view.

Álvarez, C. C. B. (2012). Riesgo psicosocial intralaboral y "burnout" en docentes universitarios de algunos países latinoamericanos. Cuadernos de Administración, 28(48), 117-132.

Araújo, T. M. (1999). Trabalho e distúrbios psíquicos em mulheres trabalhadoras de enfermagem. Tese de Doutorado em Saúde Pública. Universidade Federal da Bahia. Salvador, BA.

Araújo, T. M., Graça, C. C., \& Araújo, E. (2003). Estresse ocupacional e saúde: contribuições do Modelo Demanda-Controle. Ciência \& Saúde Coletiva, 8(4), 991-1003. DOI: 10.1590/S1413-81232003000400021.

Areias, M. E. Q., \& Guimarães, L. A. M. (2004). Gênero e estresse em trabalhadores de uma universidade pública do Estado de São Paulo. Psicologia em Estudo, 9(2), 255-262. DOI: 10.1590/S1413-73722004000200011.

Borges, L. O., Guimarães, L. A. M.,\& Silva, S. S. (2013). Diagnóstico e promoção da saúde psíquica no trabalho. In Borges, L. O. \& Mourão, L., O trabalho e as organizações: atuações a partir da psicologia (pp. 580-618). Porto Alegre: Artmed.

Brant, L. C., \& Minayo-Gomes, C. (2004). A transformação do sofrimento em adoecimento: do nascimento da clínica à psicodinâmica do trabalho. Ciência \& Saúde Coletiva, 9(1), 2013-223. DOI: 10.1590/S1413-81232004000100021.

Costa, L. S., \& Santos, M. (2013). Fatores Psicossociais de Risco no Trabalho: Lições Aprendidas e Novos Caminhos. International Journal on Working Conditions, 1(5), 3958. 
RISCOS PSICOSSOCIAIS NO TRABALHO: CONCEITOS, VARIÁVEIS E INSTRUMENTOS DE

PESQUISA

Dejours, C., \& Abdoucheli, E. (1993). Itinerário teórico em psicopatologia do trabalho. In Dejours, C.; Abdoucheli, E. \&Jayet, C., Psicodinâmica do trabalho: contribuições da escola dejouriana à análise da relação prazer, sofrimento e trabalho (pp. 119-145). São Paulo: Atlas.

Dejours, C. (2004). Addendum - da Psicopatologia à psicodinâmica do trabalho. In: Lancman, S. \& Sznelwar, L. I. (orgs.) Cristophe Dejours: Da psicopatologia à psicodinâmica do trabalho. Rio de Janeiro: Editora Fiocruz, Brasília: Paralelo.

Glina, D. M. R., Rocha, L. E., Batista, M. L.,\& Mendonça, M. G. V. (2001). Saúde mental e trabalho: uma reflexão sobre o nexo com o trabalho e o diagnóstico, com base na prática. Cad. Saúde Pública, 17(3), 607-616. DOI: 10.1590/S0102-311X2001000300015.

Gollac, M., \& Bodier M. (2011). Mesurerles facteurs psychosociaux de risque au travail pour les maîtriser (Relatório do Collège d'Expertise surle Suivides Risques Psychosociauxau Travail). Disponível em: http://www.collegerisquespsychosociauxtravail.fr/rapport-final,fr,8,59.cfm.pdf.

Guimarães, L. A. M. (2013). Fatores Psicossociais de Risco no Trabalho. In Ferreira, J. J. \& Penido, L. O., Saúde mental no trabalho: coletânea do fórum de saúde e segurança no trabalho do Estado de Goiás (pp. 273-282). Goiânia: Cir Gráfica.

Guimarães, L. A. M.,\& MacFadden, M. A. J. (1999). Validação para o Brasil do SWS@ Survey - Questionário sobre estresse, saúde mental e trabalho. In L. A. M. Guimarães \& S. Grubits (Orgs.), Série Saúde Mental e Trabalho V.1 (pp.189-208). Casa do Psicólogo: São Paulo.

Hirata, H. (2011). Tendências recentes da precarização social e do trabalho: Brasil, França, Japão. Caderno CRH, 24(1), 15-22. DOI: 10.1590/S0103-49792011000400002.

Karasek, R. A. (1979). Job Demand, job decision latitude, and mental strain: implications for job redesign. Administrative Science Quarterly, 24(2) 285-308. DOI: 10.2307/2392498.

Karasek, R. A. (1985). Job Content Questionnaire and User's Guide. Lowell (MA): University of Massachusetts.

Karasek, R. A. (1998). El modelo de Demandas-Control: enfoque social, emocional y fisiologico del risco de estres y desarrollo de comportamientos activos. In Organización Internacional Del Trabajo (OIT). Enciclopedia de Salud y Seguridade nel Trabajo (pp. 34.6-34.16). Genebra: OIT. 
Karasek, R. A.,\& Theorell, T. (1990). Healthy work: stress, productivity and there construction of working life. New York: Basic Books.

Mendes, A. M., \& Ferreira, M. C. (2007). Inventário sobre Trabalho e Riscos de Adoecimento - ITRA: Instrumento auxiliar de diagnóstico de indicadores críticos no trabalho. In Mendes, A. M. B. (org.), Psicodinâmica do trabalho: teoria, método e pesquisa (pp. 111126). São Paulo: Casa do Psicólogo.

Mendes, A. M. (2007). Da Psicodinâmica à Psicopatologia no Trabalho. In Mendes, A. M. B. (org.), Psicodinâmica do trabalho: teoria, método e pesquisa (pp. 29-48). São Paulo: Casa do Psicólogo.

Ministerio de Empleo y Seguridad Social de la España. (2012). Guía de actuaciones de la inspección de trabajo y Seguridade social sobre riesgos psicosociales. Madrid: Lerko Print S.A.

Papaioannou, I., \& Bilias, D. (2012). Psychosocial Risks at Work. Working and Living Environmental Protection, 9(1), 53-59.

Serafim, A da C., Campos, I. C. M., Cruz R. M.,\& Rabuske, M. M. (2012). Riscos psicossociais e incapacidade do servidor público: um estudo de caso. Psicologia: Ciência e Profissão, 32(3), 686-705. DOI: 10.1590/s1414-98932012000300013.

Siegrist, J. (1996). Adverse health effects of high-effort/low-reward conditions. J Occup Health Psychol, 1(1), 27-41.

Siegrist, J. (2012). Effort-reward imbalance at work -theory, measurement and evidence. Department of Medical Sociology. Disponível em: http://www.uniklinikduesseldorf.de/fileadmin/Datenpool/einrichtungen/institut_fuer_medizinische_soziologie _id54/ERI/ERI-Website.pdf. DOI: 10.1037/1076-8998.1.1.27.

Silva, C. F. (2006). Copenhagen Psychosocial Questionnaire. Fundação para a Ciência e para a Tecnologia. Análise Exacta: Portugal.

Silva, L. S., \& Barreto, S. M. (2010). Adaptação transcultural para o português brasileiro da escala effort-reward imbalance: um estudo com trabalhadores de banco. Rev Panam Salud Publica, 27(1), 32-36. DOI: 10.1590/S1020-49892010000100005

\section{As autoras:}

Aline Jacinto, mestra em Psicologia pelo Programa de Pós Graduação em Psicologia da Universidade Federal de Santa Catarina - (UFSC), especialização em Avaliação Psicológica pela Celer Faculdades - Xaxim SC, 
RISCOS PSICOSSOCIAIS NO TRABALHO: CONCEITOS, VARIÁVEIS E INSTRUMENTOS DE

PESQUISA

graduação em Psicologia pela Universidade do Vale do Itajaí -UNIVALI. Telefone: (47) 9967-7966. Email: aline-j@hotmail.com. Endereço postal: Rua Paulo Ney Laurentino, 312. Bairro Nossa Sra. Das Graças. Cidade Navegantes, SC - Brasil. CEP: 88371-211.

Suzana da Rosa Tolfo, professora associada do Departamento de Psicologia da UFSC, do Curso de Graduação em Psicologia e dos Programas de Pós-Graduação em Psicologia e em Administração da UFSC. E-mail: srtolfo14@gmail.com.

Endereço postal: Universidade Federal de Santa Catarina, Centro de Filosofia e Ciências Humanas, Departamento de Psicologia. Campus Universitário - CFH -Departamento de Psicologia - s. 12. Bairro Tindade. Cidade Florianópolis, SC - Brasil. CEP: 88040970.

Recebido em: 06/03/2017.

Aprovado em: 16/06/2017. 